\title{
Safely reducing haemodialysis frequency during the COVID-19 pandemic
}

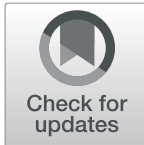

Michelle Da Silva Lodge, Thilini Abeygunaratne, Helen Alderson, Ibrahim Ali, Nina Brown, Constantina Chrysochou, Rosie Donne, Ibi Erekosima, Philip Evans, Emma Flanagan, Simon Gray, Darren Green, Janet Hegarty, Audrey Hyde, Philip A. Kalra, Elizabeth Lamerton, David Lewis, Rachel Middleton, David New, Robert Nipah, Donal O'Donoghue, Edmond O'Riordan, Dimitrios Poulikakos, Francesco Rainone, Maharajan Raman, James Ritchie, Smeeta Sinha, Grahame Wood and J. Tollitt ${ }^{*}$ (D)

\begin{abstract}
Background: Patients undergoing haemodialysis (HD) are at higher risk of developing worse outcomes if they contract COVID-19. In our renal service we reduced HD frequency from thrice to twice-weekly in selected patients with the primary aim of reducing COVID 19 exposure and transmission between HD patients.

Methods: Dialysis unit nephrologists identified 166 suitable patients (38.4\% of our HD population) to temporarily convert to twice-weekly haemodialysis immediately prior to the peak of the COVID-19 pandemic in our area. Changes in pre-dialysis weight, systolic blood pressure (SBP) and biochemistry were recorded weekly throughout the 4-week project. Hyperkalaemic patients (serum potassium $>6.0 \mathrm{mmol} / \mathrm{L}$ ) were treated with a potassium binder, sodium bicarbonate and received responsive dietary advice.
\end{abstract}

Results: There were 12 deaths (5 due to COVID-19) in the HD population, 6 of which were in the twice weekly HD group; no deaths were definitively associated with change of dialysis protocol. A further 19 patients were either hospitalised and/or developed COVID-19 and thus transferred back to thrice weekly dialysis as per protocol. 113 (68.1\%) were still receiving twice-weekly HD by the end of the 4-week project. Indications for transfer back to thrice weekly were; fluid overload (19), persistent hyperkalaemia (4), patient request (4) and compliance (1). There were statistically significant increases in SBP and pre-dialysis potassium during the project.

Conclusions: Short term conversion of a large but selected HD population to twice-weekly dialysis sessions was possible and safe. This approach could help mitigate COVID-19 transmission amongst dialysis patients in centres with similar organisational pressures.

Keywords: COVID-19, Haemodialysis, Coronavirus, Mortality, Twice weekly, SARS-CoV-2

\section{Background}

Severe Acute Respiratory Syndrome Coronavirus-2 has developed into a worldwide pandemic, with over 44 million documented cases and 1.1 million deaths worldwide. Medical comorbidities such as hypertension, diabetes mellitus, asthma, obesity and chronic kidney disease are reported as

\footnotetext{
* Correspondence: James.Tollitt@srft.nhs.uk
}

Department of Renal Medicine, Salford Royal NHS Trust, Stott Lane, Salford M68HD, UK significant predictors of morbidity and mortality in COVID-19 patients [1, 2].

The necessary frequency of haemodialysis (HD) is particularly pertinent at a time of a worldwide pandemic [3]. There is no randomised study demonstrating a beneficial effect of thrice weekly dialysis over twice weekly dialysis. HD patients are an "at-risk" group who have worse outcomes if they contract COVID-19 [4-6]. UK Renal Registry data up to 7th October 2020 has reported

(c) The Author(s). 2020 Open Access This article is licensed under a Creative Commons Attribution 4.0 International License, which permits use, sharing, adaptation, distribution and reproduction in any medium or format, as long as you give appropriate credit to the original author(s) and the source, provide a link to the Creative Commons licence, and indicate if changes were made. The images or other third party material in this article are included in the article's Creative Commons licence, unless indicated otherwise in a credit line to the material. If material is not included in the article's Creative Commons licence and your intended use is not permitted by statutory regulation or exceeds the permitted use, you will need to obtain permission directly from the copyright holder. To view a copy of this licence, visit http://creativecommons.org/licenses/by/4.0/ The Creative Commons Public Domain Dedication waiver (http://creativecommons.org/publicdomain/zero/1.0/) applies to the data made available in this article, unless otherwise stated in a credit line to the data. 
21.1\% mortality for in centre HD patients who suffer COVID-19 [7]. Reducing frequency of dialysis for some patients who dialyse in-centre may minimise patient exposure to COVID-19, allow extra space between patients undergoing dialysis and help manage unprecedented HD staff sickness [8]. The counterpoint argument follows that dialysis reduction in patients with multimorbidity may increase overall morbidity, cardiovascular events and death, especially because of the longer interdialytic gap. This gap is notorious for being associated with higher risk of death and hospitalisation even in thrice weekly patients [9-11].

Dialysis reduction in selected patients was performed in our centre prior to the peak incidence of the COVID19 pandemic in the North West of England, UK. The primary aim was to reduce COVID 19 exposure, transmission between patients and allow for social distancing whilst on the HD unit. It also permitted the formation of a 'hot' dialysis unit to cohort all suspected, proven cases and contacts for COVID-19, whereas non-exposed patients could be grouped into 'cold' satellite dialysis units. At the onset of this project we began collecting data in a structured way and this paper describes our methodology, outcomes and learning.

\section{Methods}

Our regional renal service has a catchment population of 1.55 million people and undertakes in centre haemodialysis for 432 patients in one main centre and 4 outlying satellite centres. The furthest distance between the main centre and satellite centres is 18 miles, which facilitated re-designation of patients without excessive travel times or major inconvenience. In centre HD patients were remotely reviewed (using electronic dialysis care records) by their own nephrologists to determine suitability for twice weekly dialysis (Fig. 1). No definitive inclusion criteria were specified but only named patients dialysis consultants were asked to consider suitability for twice weekly based upon their review of interdialytic weight gain, pre dialysis blood pressure and potassium, residual renal function, comorbidity and functional status. Patient concerns were addressed by the dialysis unit managers and by telephone review with their nephrologist to ensure shared decision making. All patients provided verbal consent to be included within this study. Exclusion criteria included; 1) already receiving twice weekly dialysis; 2) patient refusal 3) highly irregular attendance for dialysis 4) hospitalised on the 23rd March 2020. Patients were transferred to twice weekly dialysis at the beginning of the week commencing 23rd March 2020 and received telephone dietary and fluid advice regarding potential changes in fluid and food intake which may have been necessary. All twice weekly dialysis took place in the satellite centres which were maintained as 'cold' sites, whilst the main centre was designated the 'hot site' for dialysis of suspected and confirmed COVID-19 patients. The 'hot' site also grouped HD patients who were contacts within a COVID-19 household. Patients underwent structured active monitoring of their dialysis parameters between Monday 23rd March 2020

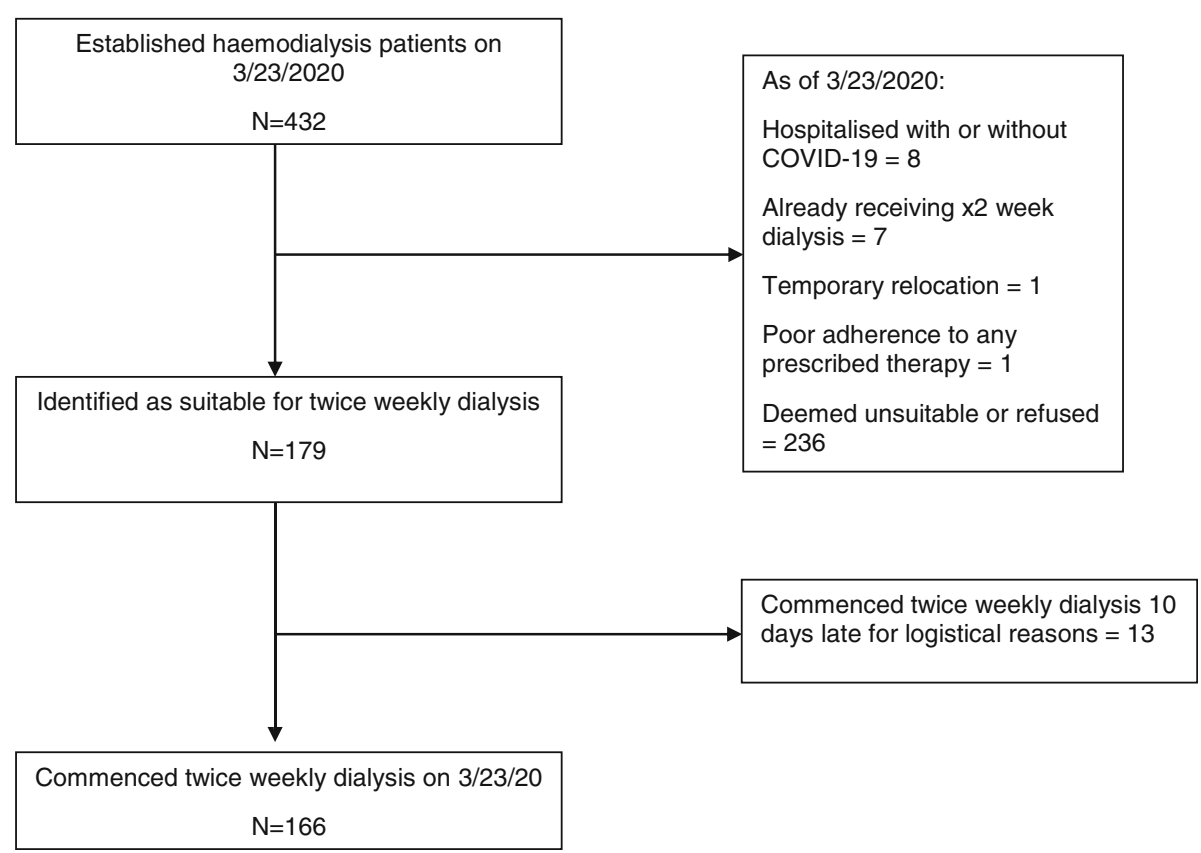

Fig. 1 Consort diagram of patient participation 
and Monday 20th April 2020. Target weight, pre-dialysis systolic blood pressure (SBP) and pre-dialysis potassium were taken from the first dialysis session of the week preceding conversion for comparative purposes. During week $1(3 / 23 / 20$ to $3 / 29 / 20)$ patients were dialysed on Monday and Friday if their usual days were Monday, Wednesday and Friday. Similarly, patients who dialysed on Tuesday, Thursday, Saturday were dialysed on Tuesday and Saturday. During week 2, 3 and 4 patients were dialysed on Monday and Thursday, Tuesday and Friday or Wednesday and Saturday. Dialysis parameters were remotely reviewed at the end of each day for the first dialysis session of each week. Key safety indicators included pre-dialysis SBP, pre-dialysis weight gain, ultrafiltration rate (UFR) and pre-dialysis potassium $(\mathrm{K}+)$. Two nephrologists (JT + IE or IA or AH or PE) independently reviewed each patient's dialysis data and agreed interventions. A pre-dialysis $\mathrm{K}+$ of $\geq 6.5 \mathrm{mmol} / \mathrm{L}$ was the only absolute indication to convert back to thrice weekly dialysis. Increased surveillance of dialysis parameters were indicated if any of the following events occurred: SBP > $180 \mathrm{mmHg}$, UFR $>10 \mathrm{~mL} / \mathrm{kg} / \mathrm{hr}$., pre-dialysis weight > $5 \%$ of target weight and $>31$ ultrafiltration (UF) per session. Dietetic consultation and medical review of both dialysis sessions in that week were undertaken. Predialysis potassium was monitored closely with the following management plan adopted:

\section{Pre-dialysis potassium $(\mathrm{mmol} / \mathrm{l})$}

$5.7-6.0$

$6.1-6.4$

\section{Action}

Repeat pre-dialysis potassium at next dialysis session alongside dietetic telephone consultation

Commence potassium binder ( $5 \mathrm{~g}$ once a day of sodium zirconium cyclosilicate [12]), sodium bicarbonate $1 \mathrm{~g} 3$ times per day [13] and dietetic telephone consultation. Repeat predialysis potassium at next dialysis session. If potassium $>6.0$ at next session increase dose of binder by $5 \mathrm{~g}$ OD to maximum $15 \mathrm{~g}$ OD.
Renal pharmacists and renal dietitians prescribed and managed the prescriptions, patient counselling and hyperkalaemia dietary advice. Dialysis prescriptions (including dialysate potassium) were not changed during this period. All patients were prescribed $4 \mathrm{~h}$ of dialysis.

Demographic, comorbidity, biochemical and dialysis adequacy comparisons between patients who continued to receive thrice weekly dialysis and those transferred to twice weekly dialysis were performed. Diagnosis, medication and comorbidity data were taken from coded diagnoses available on hospital and primary care electronic medical records. Usual pre-dialysis blood pressures were calculated using the average of the last readings prior to $3 / 23 / 20$. Urine output was not contemporaneously measured but patients who selfreported anuria $(<100 \mathrm{~mL}$ per day) was recorded. Thirteen patients who commenced twice weekly dialysis after being identified later on during the course of this project were subsequently excluded from analysis in the thrice weekly group at baseline.

Patients who were hospitalised or admitted to the COVID-19 dialysis 'hot unit' were transferred to thrice weekly dialysis for the rest of the project. All hospitalisations were recorded after scrutinizing hub hospital (where the main centre is based) admission data and all referrals from surrounding district hospitals for the period $3 / 23 / 20$ to $4 / 20 / 20$. Patients were defined as COVID-19 positive after a positive nasal and/or throat COVID-19 PCR. Patients who were transferred back to thrice weekly dialysis completed 3 dialysis sessions in the following 7 days. Cause of death was obtained from death certification and all fatal cases were discussed at a weekly multi-disciplinary mortality and morbidity meeting (including at least 5 nephrologists) to evaluate whether the reduction in dialysis frequency was contributory.

This is an observational report of a strategic and organisational restructuring of dialysis provision in our centre during the COVID 19 pandemic..

\section{Statistical analysis}

Data were analysed using means and standard deviations (parametric data) and medians and interquartile ranges (non-parametric data) where appropriate. Categorical data were compared using chi square test. Continuous data were compared using unpaired T test and Mann U Whitney. Significance of pre-dialysis SBP, potassium and weight changes were compared at each data point (after week1, after week 2 and after week 3) using MannWhitney $U$ test. Data were analysed on an as-treated basis. Only patients participating in twice weekly dialysis at each time point were analysed.

\section{Results}

\section{Baseline characteristics}

From a cohort of 432 in-centre dialysis patients 179 $(41.4 \%)$ were identified as potentially suitable for and agreed to undergo twice weekly dialysis. This manuscript reports on the 166 (38.4\%) patients who then commenced twice weekly dialysis on $3 / 23 / 20$. Patients who were transferred to twice weekly dialysis were more likely to be older, with lower ultrafiltration volumes, greater urea reduction ratio (URR), shorter dialysis vintage and to have lower pre-dialysis phosphate and potassium levels. There was no significant difference in the frequency of primary renal diseases between the two groups and there were significantly less patients with heart failure transferred to twice weekly HD (Table 1). 
Table 1 Baseline clinical and laboratorial characteristics of HD patients

\begin{tabular}{|c|c|c|c|}
\hline & Twice weekly HD $(n=166)$ & Thrice weekly HD $(n=236)$ & $P$ value \\
\hline Age (years) & $65.0[54.8-74.3]$ & $59.0[49.0-71.0]$ & $<0.001$ \\
\hline Sex- Male (\%) & $107(64.5)$ & $154(65.3)$ & 0.870 \\
\hline Ethnicity - White British (\%) & $110(66.3)$ & $167(70.8)$ & 0.339 \\
\hline Pre HD weight $(\mathrm{Kg})^{\mathrm{a}}$ & $73.5[62.0-85.3]$ & $76.2[64.2-91.4]$ & 0.038 \\
\hline$B M I^{b}$ & $25.8[22.9-29.4]$ & $27.5[23.3-31.6]$ & 0.050 \\
\hline Last URR $(\%)^{c}$ & $71.1[65.4-75.5]$ & $69.3[63.1-74.4]$ & 0.009 \\
\hline Corrected Calcium ${ }^{d}$ (mmol/L) & $2.36 \pm 0.18$ & $2.34 \pm 0.17$ & 0.283 \\
\hline Phosphate $(\mathrm{mmol} / \mathrm{L})$ & $1.56[1.27-1.89]$ & $1.68[1.33-2.07]$ & 0.023 \\
\hline Parathyroid Hormone ${ }^{f}(\mathrm{pmol} / \mathrm{L})$ & $26.9[12.5-56.8]$ & 28.9 [13.2-51.9] & 0.361 \\
\hline Haemoglobin ${ }^{9}$ (g/L) & $107 \pm 16.1$ & $107 \pm 17.6$ & 0.873 \\
\hline Potassium $^{\mathrm{h}}(\mathrm{mmol} / \mathrm{L})$ & $4.50[4.00-5.20]$ & $4.90[3.20-5.50]$ & $<0.001$ \\
\hline Albumin ${ }^{\mathrm{i}}(\mathrm{g} / \mathrm{L})$ & $38.0[34.1-41.0]$ & $38.5[35.0-41.0]$ & 0.358 \\
\hline Pre-HD average SBPj & $148 \pm 21.7$ & $147 \pm 22.4$ & 0.617 \\
\hline Pre-HD average DBP ${ }^{k}$ & 74.0 [62.3-84.8] & 77.5 [67.0-88.0] & 0.082 \\
\hline Post-HD average SBP' & 139 [126-154] & $137[121-156]$ & 0.980 \\
\hline Post-HD average DBPm & $72.0[63.0-80.0]$ & 71.5 [63.8-83.3] & 0.584 \\
\hline Average $\mathrm{UF}^{\mathrm{n}}(\mathrm{L})$ & $1.40[1.00-2.00]$ & $2.00[1.50-2.70]$ & $<0.001$ \\
\hline Anuric Status $^{\circ}(\mathrm{n})$ & 16 & N/A & \\
\hline Dialysis Vintage (months) & $21(6.3-14.5)$ & $29.5(11.9-261)$ & 0.013 \\
\hline \multicolumn{4}{|l|}{ Primary Renal Disease } \\
\hline Diabetic Nephropathy (\%) & $51(30.7)$ & $81(34.3)$ & 0.450 \\
\hline Hypertensive/Renovascular disease (\%) & $27(16.3)$ & $30(12.7)$ & 0.314 \\
\hline Glomerulonephritis (\%) & $21(12.7)$ & $31(13.1)$ & 0.886 \\
\hline ADPKD (\%) & $15(9.0)$ & $13(5.5)$ & 0.172 \\
\hline Urological (\%) & $13(7.8)$ & $24(10.2)$ & 0.425 \\
\hline Pyelonephritis (\%) & $3(1.8)$ & $11(4.7)$ & 0.125 \\
\hline ANCA-associated vasculitis (\%) & $4(2.4)$ & $5(2.1)$ & 0.847 \\
\hline Other (\%) & $17(10.2)$ & $16(6.8)$ & 0.214 \\
\hline Unknown (\%) & $15(9.0)$ & $25(10.6)$ & 0.610 \\
\hline \multicolumn{4}{|l|}{ Comorbidity } \\
\hline Ischaemic heart disease (\%) & $25(15.1)$ & $40(17.0)$ & 0.613 \\
\hline Heart Failure (\%) & $5(3.0)$ & $18(7.6)$ & 0.049 \\
\hline CVA (\%) & $16(9.7)$ & 35 (14.8) & 0.124 \\
\hline Diabetes Mellitus (\%) & $62(37.4)$ & $101(42.8)$ & 0.274 \\
\hline ACEi (\%) & $30(18.1)$ & $46(19.5)$ & 0.721 \\
\hline ARB (\%) & 19 (11.5) & 32 (13.6) & 0.532 \\
\hline
\end{tabular}

Results are expressed as mean $\pm \mathrm{SD}$, median [IQR; interquartile range] or $\mathrm{n}(\%) \cdot p$-value calculated using unpaired $\mathrm{T}$ test for parametric data and Mann-Whitney $\mathrm{U}$ Test for non-parametric data. Categorical variables were analysed by Chi-square test. Abbreviations: BMI - Body Mass Index, HD - Haemodialysis, SBP - Systolic Blood Pressure, DBP - Diastolic Blood Pressure, URR - Urea Reduction Ratio, ADPKD - Autosomal Dominant Polycystic Kidney Disease, CVA - Cerebrovascular event, ACEi- Angiotensin converting enzyme inhibitor, ARB - Angiotensin receptor blocker. ${ }^{a}$ missing for 2 patients in twice weekly group. ${ }^{\mathrm{b}}$ missing for 2 patients in twice weekly group and 4 patients in thrice weekly group. ' $m i s s i n g$ for 26 patients in twice weekly group and 38 patients in thrice weekly group. ${ }^{d}$ missing for 2 patients in twice weekly group. ${ }^{\mathrm{e}}$ missing for 2 patients in twice weekly group. ${ }^{\mathrm{f}}$ missing for 4 patients in twice weekly group and 78 patients in thrice weekly

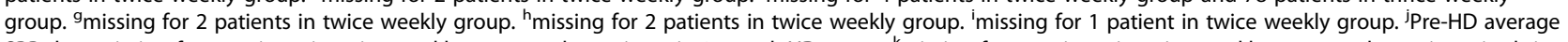
SBP data missing for 4 patients in twice weekly group and 4 patients in 3 x week HD group. ${ }^{k}$ missing for 4 patients in twice weekly group and 4 patients in thrice weekly group. 'missing for 9 patients in twice weekly group and 10 patients in thrice weekly group. ${ }^{\mathrm{m}}$ Post-HD average DBP data missing for 9 patients in twice weekly group and 10 patients in thrice weekly group. ${ }^{n}$ missing for 3 patients in twice weekly group. ${ }^{\circ}$ missing data 79 patients 


\section{Longitudinal changes in dialysis parameters}

Dialysis parameters of those patients who remained on a twice weekly dialysis regime demonstrated that predialysis weight and interdialytic percentage increase in body weight remained unchanged (Fig. 2a and b). However, as the weeks progressed there was a significant increase in median SBP and potassium for those who remained on the twice weekly dialysis regime. These findings persisted after excluding patients who were known to be anuric (data not shown). The increase in SBP was apparent after 2 weeks of twice weekly dialysis (Fig. 2c) and the median SBP at the end of 3 weeks of twice weekly dialysis was $153(140-172) \mathrm{mmHg}$ compared with a median SBP at baseline of 145 (132-165) $\mathrm{mmHg}$. The number of patients with a pre-dialysis $\mathrm{SBP}>180 \mathrm{mmHg}$ at baseline and after weeks 1,2 and 3 were $17,18,21$ and 20 , respectively.
There was a significant longitudinal increase in predialysis potassium. This difference was apparent after 1 week of twice weekly dialysis (Fig. 2d). The median potassium at the end of 3 weeks in those patients able to continue twice weekly dialysis was $4.7 \mathrm{mmol} / \mathrm{L}(4.2-5.2)$ compared with $4.5 \mathrm{mmol} / \mathrm{L}(4.1-4.9)$ at baseline. The number of patients with a pre-dialysis potassium above $6.0 \mathrm{mmol} / \mathrm{L}$ was $0,8,12$ and 6 at baseline and after 1,2 and after 3 weeks of twice weekly dialysis respectively.

\section{Technique survival}

We determined this by including patients who died (6), patients automatically transferred back to thrice weekly dialysis because of hospitalisation (13) or suspected/ confirmed COVID-19 infection (6) as "failing twice weekly dialysis". There were 113 (68.1\%) patients who were able to continue twice weekly dialysis for the whole
A - Pre-dialysis weight

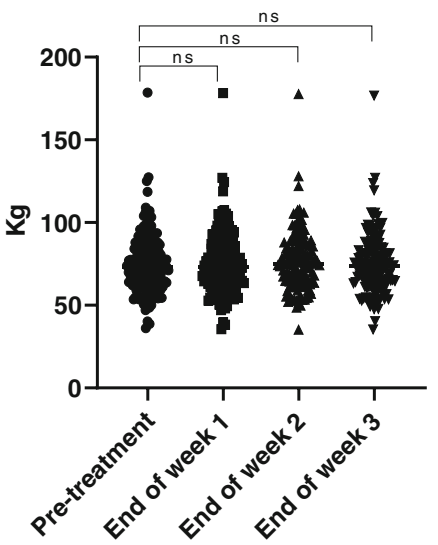

C - Pre-dialysis Systolic Blood Pressure

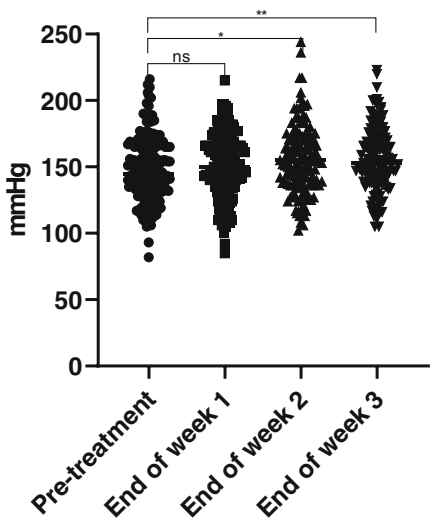

B - Percentage change in body weight

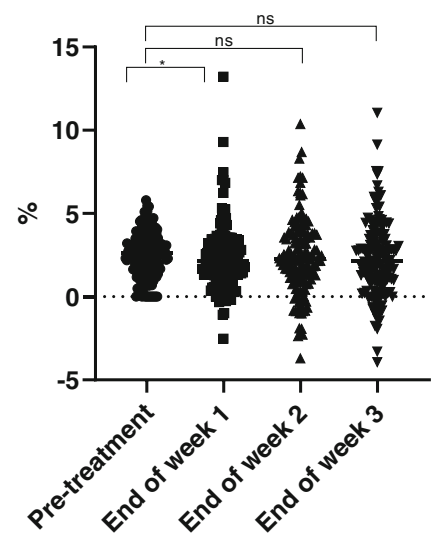

D - Pre-dialysis Potassium

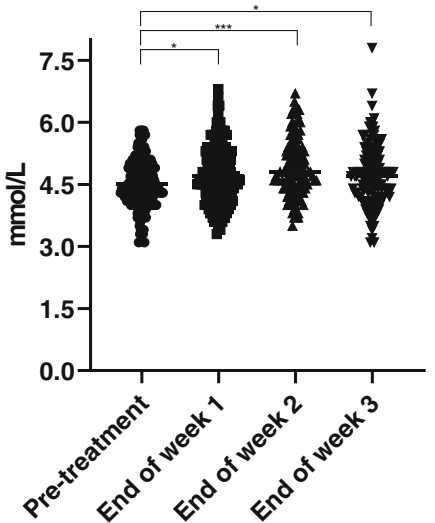

Fig. 2 Changes in dialysis parameters in HD patients who remained on twice weekly dialysis. a - Pre-dialysis weight. b - Percentage change in body weight. $\mathbf{c}$ - Pre-dialysis Systolic Blood Pressure. $\mathbf{d}$ - Pre-dialysis Potassium. Statistical significance is shown by Mann-Whitney test: ns = not statistically significant, ${ }^{*} p<0.05,{ }^{* *} p<0.01,{ }^{* *} p<0.001$ between twice and thrice weekly HD patients 
4 week period (Fig. 3). This resulted in 452 fewer dialysis sessions potentially minimising 452 potential exposures to COVID-19 both during dialysis and on transport to and from the units. There were 28 patients we electively transferred back to thrice weekly dialysis during the 4 weeks of the project. The indications for transfer back were fluid overload $(n=19)$, hyperkalaemia despite use of binders $(n=4)$, patient's request $(n=4)$ and compliance concerns $(n=1)$. The characteristics of these 28 patients who we know "failed" twice weekly dialysis due to definite dialysis related reasons (rather than exposure or contraction of COVID-19 for example) are displayed in Table 2.

\section{Other clinical outcomes}

The main outcomes for patients are displayed in Table 3. There were 12 deaths in the entire HD population during these 4 weeks, of whom 6 had been transferred to twice weekly HD. No death was directly related to twice weekly dialysis (Table 4). Two patients died from acute ischaemic events after $12 \mathrm{~h}$ but within $48 \mathrm{~h}$ of their last dialysis session, 1 patient died from COVID-19 pneumonia, whilst another died from suspected COVID-19 pneumonia. Two patients died at home $>12 \mathrm{~h}$ but $<24 \mathrm{~h}$ after their last dialysis session; there was no suspicion of COVID-19. One was frail and elderly and the other was an unexpected sudden death that was referred to the coroner. Sudden cardiac death is a common cause of death for patients undergoing dialysis [14]. An association between dialysis reduction to this sudden death was not thought likely based upon dialysis parameters and laboratory results from the preceding dialysis session.

There were proportionately less patients who suffered COVID-19 in the twice weekly group but this difference was not statistically significant. There were no admissions with fluid overload in the twice weekly group during this 4-week project.

\section{Hyperkalaemia interventions}

There were 19 patients who received a prescription of sodium bicarbonate $1 \mathrm{~g}$ thrice daily and sodium zirconium cyclosilicate alongside further dietetic advice. Two of these patients died ( 1 with normal potassium on admission and 1 with normal pre-dialysis potassium on the previous dialysis session $(4.8 \mathrm{mmol} / \mathrm{L}) .7 / 19$ patients were subsequently transferred back to thrice weekly dialysis and 1 patient did not tolerate either medication. Nine patients continued to take the medications and dialyse twice weekly. Hyperkalaemia interventions reduced pre-dialysis potassium by $0.9 \mathrm{mmol} / \mathrm{L}$ (IQR $0.8-1.4$ ); one patient's potassium increased following intervention.

\section{Discussion}

This analysis found that the majority of patients who are deemed suitable to temporarily convert to twice weekly dialysis were able to safely dialyse twice weekly for at least 1 month during the COVID-19 pandemic, enabling

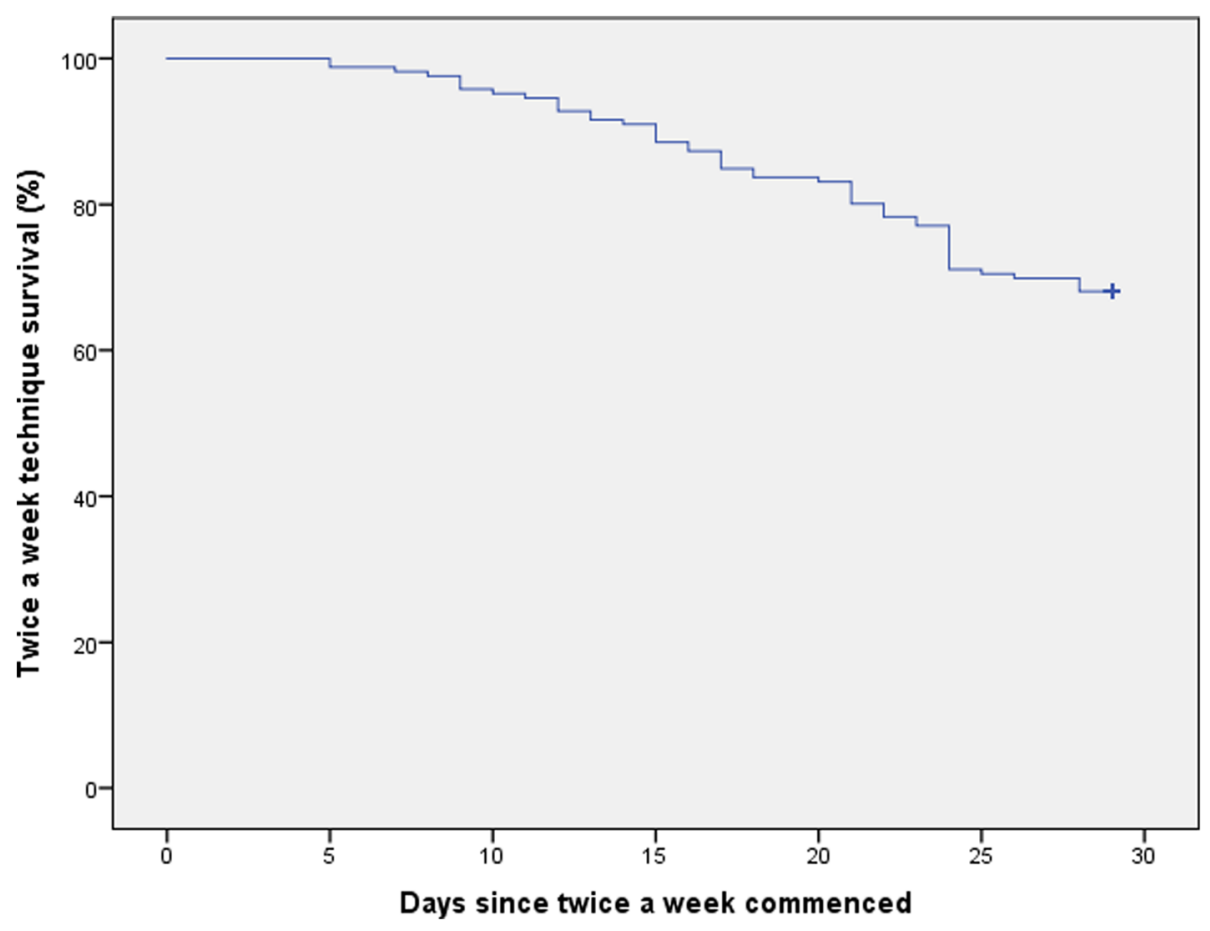

Fig. 3 Technique survival curve in eligible population 
Table 2 Main clinical outcomes of the project at 4 weeks after treatment change

\begin{tabular}{llll}
\hline & Twice weekly HD & Thrice weekly HD & P value \\
\hline${\text { URR }(\%)^{\mathrm{a}}}$ & $72.6[66.4-77.2]$ & $69.7[62.7-74.8]$ & $\mathbf{0 . 0 0 9}$ \\
Corrected Calcium $^{\mathrm{b}}(\mathrm{mmol} / \mathrm{L})$ & $2.33[2.20-2.42]$ & $2.33[2.22-2.43]$ & 0.921 \\
Phosphate $^{\mathrm{c}}(\mathrm{mmol} / \mathrm{L})$ & $1.77[1.44]$ & $1.65[1.33-2.09]$ & 0.117 \\
Potassium $^{\mathrm{d}}(\mathrm{mmol} / \mathrm{L})$ & $4.80[4.30-5.40]$ & $4.90[4.40-5.40]$ & 0.329 \\
UF per session (L) & $1.50[1.00-2.03]$ & $2.00[1.40-2.63]$ & $<0.001$ \\
COVID-19 positive (\%) & $9(5.4)$ & $20(8.5)$ & 0.245 \\
Hospitalisations (\%) & $13(7.8)$ & $17(7.2)$ & 0.813 \\
Deaths (\%) & $6(3.6)$ & $6(2.5)$ & 0.535 \\
\hline
\end{tabular}

Results are expressed as median [IQR; interquartile range] or $\mathrm{n}$ (\%). p-value calculated using Mann-Whitney U Test. Categorical variables were analysed by Chisquare test. Hospitalisation data includes patients who were hospitalised for COVID-19 and prior to death. ${ }^{\mathrm{a}}$ missing for 56 patients in twice weekly group and 126 patients in thrice weekly group. ${ }^{b}$ missing for 9 patients in twice weekly group and 46 patients in thrice weekly group. ${ }^{c}$ missing for 9 patients in twice weekly group and 46 patients in thrice weekly group. ${ }^{d}$ missing for 32 patients in thrice weekly group

COVID-19, hospitalisations and deaths \% calculated in relation to original number of patients $n=166 / n=236$. Abbreviations: URR Urea reduction ratio, UF Ultrafiltration

safer grouping of patients to reduce potential viral exposure and transmission and ease service demands which may have been exacerbated by staff sickness. However, this was only possible with very close monitoring via dedicated clinician time and through the use of digital technology allowing remote monitoring of biochemistry and dialysis parameters. The necessity for close monitoring can be demonstrated for two reasons. Firstly, longitudinal assessment of dialysis parameters demonstrated statistically significant increases in predialysis systolic blood pressures and pre-dialysis potassium in those patients who continued to receive twice weekly dialysis, although overall these parameters remained well within 'safe' limits. Secondly, it was also noted that the rate of patient transfer back to thrice weekly dialysis was constant throughout the project at around 3-4\% per week.

Rising SBP despite no significant increase in predialysis weight suggests that blood pressure changes were not necessarily related to increases in extracellular blood volume (ECV). This finding is not surprising given that more frequent dialysis has been shown to improve blood pressure control through various mechanisms. These include reduced ECV, increased sodium removal, reduced sympathetic tone and removal of vasoactive factors which may be driving hypertension [15-17]. There were similar numbers of patients with a pre-dialysis $\mathrm{SBP}>180 \mathrm{mmHg}$ before the project commenced compared with at the end of this twice weekly dialysis project (17 versus 20). A value of $\mathrm{SBP}>180 \mathrm{mmHg}$ as a trigger for closer dialysis parameter observation and possible conversion back to thrice weekly after the next dialysis session was based upon evidence that this value delineates an increased mortality risk in dialysis patients, although this evidence is conflicting [18].

There was little difference in the UF volumes in the twice weekly patients at baseline compared with those still maintaining on the twice weekly protocol at the end of the 4-week period (1.4 (1.0-2.0) litres per session compared with 1.5 (1.0-2.0) litres per session). However, this excludes the 19 (13.5\%) patients who were transferred back to thrice weekly because of fluid-related issues. Table 3 demonstrates that patients with higher ultrafiltration at baseline were those who could not manage twice weekly dialysis for a 4 week period.

Due to reduced weekly dialysis time it is unsurprising that the median pre-dialysis potassium significantly increased every week. A pre dialysis potassium $>6.0$ $\mathrm{mmol} / \mathrm{L}$ has been suggested as a threshold whereby mortality risk substantially increases [19]. However only 6 patients had a pre-dialysis potassium above $6.0 \mathrm{mmol} /$ $\mathrm{L}$ and $75 \%$ of the patients had a pre-dialysis potassium $<$ $5.4 \mathrm{mmol} / \mathrm{L}$ at the end of this project. This was the same as in the thrice weekly population. Only 2 of the 6 patients with this degree of hyperkalaemia had previously had a pre-dialysis potassium above $6.0 \mathrm{mmol} / \mathrm{L}$ during the entire project. We had made no changes to dialysate potassium concentrations because recent evidence has suggested a higher mortality risk when patients are dialysed against a low potassium dialysate $(1 \mathrm{mEql} / \mathrm{l})$, particularly those patients with a higher serum potassium [20]. The use of potassium binders, sodium bicarbonate and responsive dietetic consultations mitigated against the need to increase dialysis session frequency in 9 patients. The role of potassium binders to reduce hyperkalaemia events and major adverse cardiovascular events in dialysis patients has not been researched in any randomised control study [12]. Potassium profiling was not used in this study but could be an option to mitigate hyperkalaemia in centres where this is an option [21].

There were no significant differences between hospitalisations, COVID-19 infections and deaths between the two groups although the twice weekly group were on average 6 years older than the thrice weekly 
Table 3 Baseline clinical and laboratorial characteristics for twice weekly patients

\begin{tabular}{|c|c|c|c|}
\hline & Completed full trial $(n=113)$ & Failed twice weekly dialysis $(n=28)$ & $P$ value \\
\hline Age (years) & $63.1 \pm 14.2$ & $65.1 \pm 14.7$ & 0.5257 \\
\hline Sex- Male (\%) & $76(67.3)$ & $12(52.2)$ & 0.1686 \\
\hline Ethnicity - White British (\%) & $78(69.0)$ & $17(73.9)$ & 0.6420 \\
\hline Pre HD weight (Kg) & $73.5[62.0-83.2]$ & $76.4[71.6-86.6]$ & 0.1545 \\
\hline BMI & $25.8[22.7-28.8]$ & $28.2[24.5-32.9]$ & 0.0395 \\
\hline Last URR $(\%)^{a}$ & $70.6[66.2-76.7]$ & $72.5[69.3-74.3]$ & 0.8383 \\
\hline Corrected Calcium (mmol/L) & $2.35 \pm 0.18$ & $2.36 \pm 0.18$ & 0.7784 \\
\hline Phosphate (mmol/L) & $1.55 \pm 0.44$ & $1.68 \pm 0.56$ & 0.2030 \\
\hline Parathyroid Hormone ${ }^{\mathrm{b}}$ (pmol/L) & $28.3[12.0-59.2]$ & $25.7[11.6-36.5]$ & 0.3732 \\
\hline Haemoglobin (g/L) & $108 \pm 15.5$ & $101 \pm 19.9$ & 0.0940 \\
\hline Potassium (mmol/L) & $4.52 \pm 0.77$ & $4.76 \pm 0.84$ & 0.1792 \\
\hline Albumin $(g / L)$ & $38.3 \pm 4.65$ & $35.0[32.7-40.0]$ & 0.0557 \\
\hline Pre-HD average SBP & $148 \pm 21.3$ & $149 \pm 21.0$ & 0.8362 \\
\hline Pre-HD average DBP & $73.9 \pm 15.4$ & $74.8 \pm 13.1$ & 0.7889 \\
\hline Post-HD average SBP & 138 [125-155] & 134 [125-150] & 0.5987 \\
\hline Post-HD average DBPd & $71.0[62.5-80.0]$ & $72.0[64.0-79.0]$ & 0.8243 \\
\hline Average UF (L) & $1.30[1.00-2.00]$ & $2.20[1.40-2.60]$ & 0.0007 \\
\hline Dialysis Vintage (months) & $19.2(5.3-45.2)$ & $20.7(8.0-61.4)$ & 0.5270 \\
\hline \multicolumn{4}{|l|}{ Primary Renal Disease } \\
\hline Diabetic Nephropathy (\%) & $35(31.0)$ & $9(39.1)$ & 0.4508 \\
\hline Hypertensive/Renovascular disease (\%) & $20(17.7)$ & $4(17.4)$ & 0.9727 \\
\hline Glomerulonephritis (\%) & $13(11.5)$ & $5(21.7)$ & 0.1897 \\
\hline ADPKD (\%) & $11(9.7)$ & $2(8.7)$ & 0.8821 \\
\hline Urological (\%) & $9(8.0)$ & $1(4.3)$ & 0.5375 \\
\hline Pyelonephritis (\%) & $2(1.8)$ & $0(0.0)$ & 0.5183 \\
\hline ANCA-associated vasculitis (\%) & $2(1.8)$ & $0(0.0)$ & 0.5183 \\
\hline Other (\%) & $11(9.7)$ & $0(0.0)$ & 0.1207 \\
\hline Unknown (\%) & $10(8.8)$ & $2(8.7)$ & 0.9877 \\
\hline \multicolumn{4}{|l|}{ Comorbidity } \\
\hline Ischaemic heart disease (\%) & $21(18.6)$ & $2(8.7)$ & 0.2502 \\
\hline Heart Failure (\%) & $4(3.5)$ & $1(4.3)$ & 0.8523 \\
\hline CVA (\%) & $8(7.1)$ & $6(26.1)$ & 0.0065 \\
\hline Diabetes Mellitus (\%) & $48(42.5)$ & $10(43.5)$ & 0.9298 \\
\hline ACEi (\%) & $24(21.2)$ & $5(21.7)$ & 0.9576 \\
\hline ARB (\%) & $18(15.9)$ & $0(0.0)$ & 0.0408 \\
\hline
\end{tabular}

Results are expressed as mean $\pm \mathrm{SD}$, median [IQR; interquartile range] or $\mathrm{n}(\%) . p$-value calculated using unpaired T test for parametric data and Mann-Whitney $\mathrm{U}$ Test for non-parametric data. Categorical variables were analysed by Chi-square test. Abbreviations: BMI - Body Mass Index, HD - Haemodialysis, SBP - Systolic Blood Pressure, DBP - Diastolic Blood Pressure, URR - Urea Reduction Ratio, ADPKD - Autosomal Dominant Polycystic Kidney Disease, CVA - Cerebrovascular event, ACEi- Angiotensin converting enzyme inhibitor, ARB - Angiotensin receptor blocker. ${ }^{a}$ missing for 13 patients in completed full twice weekly group and 5 patients in failed twice weekly group. ${ }^{b}$ missing for 1 patient in failed twice weekly group. ${ }^{c}$ missing for 4 patients in completed full twice weekly group. ${ }^{d}$ missing for 4 patients in completed full twice weekly group

group, perhaps representing a more at-risk group, although they had lower prevalence of heart failure. There was no definite evidence that any of the 6 deaths in the twice weekly dialysis group were caused by a reduction in dialysis frequency. There were no hospitalisations for fluid overload in the twice weekly group.

The analysis of this 4-week period of change in dialysis protocol is not intended to re-energise the debate over long-term dialysis frequency and dialysis dose but 
Table 4 Causes of death

\begin{tabular}{lll}
\hline & Twice weekly HD & Thrice weekly HD \\
\hline COVID Pneumonia & 1 & 4 \\
Cardiovascular Disease & 2 & 0 \\
Myeloma & 0 & 1 \\
Natural Causes & 0 & 1 \\
Chronic Kidney Disease/End & 2 & 0 \\
Stage Renal Disease & & \\
Uncertain (sudden death & 1 & 0 \\
at home) & \\
Total & 6 & 6
\end{tabular}

Causes of death were taken from death certification records. All deaths were discussed in mortality and morbidity meetings to ensure there was no direct relationship between dialysis reduction and cause of death. ${ }^{\mathrm{a}} \mathrm{This}$ patient has been referred for a coroner's investigation (unrelated to twice weekly dialysis)

provides a potential methodology to appropriately and safely rationalise dialysis resources during a health service crisis such as the current pandemic. Although we are not recommending generalisation of our approach as health care management differs markedly throughout the world, it may have implications for other countries where dialysis resources are limited. Although we did not have definitive inclusion criteria, we have retrospectively surveyed the clinicians involved and together with the findings of this study we would suggest that short term switch to twice weekly dialysis is most appropriate for;

- Elderly patients

- Patients with lower ultrafiltration requirements/ higher residual renal function

- Patients whose pre-dialysis phosphate levels are within the normal range

- Patients whose pre-dialysis potassium levels are well within normal range

- Patients who share the decision to switch to twice weekly dialysis

We investigated complete conversion of patients to twice weekly dialysis. Future studies could investigate the impact of alternating twice to thrice weekly dialysis in selected groups of patients to minimise COVID-19 exposure but maintain satisfactory dialysis parameters. At this stage, having passed the first peak of the COVID-19 pandemic in our geographical region, all our in-centre haemodialysis patients are now being individually reassessed for their suitability and preference for either home haemodialysis or peritoneal dialysis, the latter with particular relevance to patients with residual urine output currently treated with twice weekly haemodialysis. Patients who will not be suitable or do not wish to be transferred to home therapies and do not have significant formally quantified residual renal function will return to thrice weekly dialysis in a planned fashion over the next few weeks [22].

\section{Conclusions}

In conclusion, the COVID-19 pandemic is an international exceptional health crisis. This project demonstrates that reorganisation of dialysis provision for selected patients has the potential to protect patients and clinical staff. Patient selection and careful real-time monitoring can ensure that abrupt changes in dialysis provision are safe.

\section{Limitations}

This analysis did not fully evaluate dialysis adequacy which is reported to influence long term outcomes of HD as this project was primarily designed to overcome the short-term challenge of dialysis provision during a pandemic. It was not possible to perform contemporaneous measurements of residual renal function due to the short lead time of project set-up and there was considerable missing data regarding anuric status (47.6\%). Regular measurement of residual renal function within the whole dialysis cohort would undoubtedly aid in decision making to determine suitability to convert to twice weekly haemodialysis at short notice. There was no measure of concordance with interventions (i.e. compliance with potassium binder medication) for hyperkalaemia although all but one patient's potassium fell after its initiation. Whilst patient's views were continually monitored during this period there were no quality of life or intradialytic health related quality of life symptoms recorded throughout the 4-week period.

\section{Abbreviations \\ ACEi: Angiotensin Converting Enzyme inhibitor; ADPKD: Autosomal Dominant Polycystic Kidney Disease; ARB: Angiotensin receptor blocker; BMI: Body Mass Index; COVID-19: Coronavirus disease; CVA: Cerebrovascular event; HD: Haemodialysis; DBP: Diastolic Blood Pressure; ECV: Extracellular blood volume; IQR: Interquartile Range; $\mathrm{K}^{+}$: Potassium; OD: Once Daily; SBP: Systolic Blood Pressure; UF: Ultrafiltration; UFR: Ultrafiltration Rate;} URR: Urea Reduction Ratio

\section{Acknowledgements}

This piece of work could not have been performed without the understanding and flexibility of our renal patients. A great level of thanks must go to the renal dietetic team, pharmacy and administrative teams.

\section{Authors' contributions}

The co-authorship list is large as it includes key health care staff that were involved in the organisation of and monitoring of this service change, and also all other consultant nephrologists in our department, in recognition of their great collaborative efforts during this exceptionally challenging period. All authors have read and approved the manuscript. Concept and proof reading: $D P, P K, J T, R M, D N, J R, F R, R D, T C, T A, D L, N B, H A$, DOD, EOR, PE, IA, IE, MR, GW, SS, LL, DG, RN, SG. Data Collection: JT, IA, AH, PE, EF, IE. Data Analysis: MDSL. Manuscript: MDSL, PK, JT.

\section{Funding}

Not applicable.

Availability of data and materials

All data generated or analysed during this study are included in this published article. 


\section{Ethics approval and consent to participate}

Online tools provided by the NHS Health Research Authority determined that research ethics committee review was not required in accordance with the UK Policy Framework for Health and Social Care Research. The study was performed in accordance with the declaration of Helsinki and approval for this study, including method of consent, was obtained from the Research and Innovation department of the Northern Care Alliance NHS Group (Ref: S19HRANA34). Patients provided verbal consent to be included in this project.

\section{Consent for publication}

Not applicable.

\section{Competing interests}

Professor Kalra reports grants and personal fees from Vifor, personal fees from AstraZeneca, outside the submitted work. No other authors have anything else to declare.

Received: 14 July 2020 Accepted: 17 November 2020

Published online: 07 December 2020

\section{References}

1. Wu C, Chen X, Cai Y, Xia J, Zhou X, Xu S, et al. Risk factors associated with acute respiratory distress syndrome and death in patients with coronavirus disease 2019 pneumonia in Wuhan, China. JAMA Intern Med. 2020;180(7): 934.

2. Zhou F, Yu T, Du R, Fan G, Liu Y, Liu Z, et al. Clinical course and risk factors for mortality of adult inpatients with COVID-19 in Wuhan, China: a retrospective cohort study. Lancet. 2020;395(10229):1054.

3. Scribner $\mathrm{BH}$, Cole JJ, Ahmad S, Blagg CR. Why thrice weekly dialysis? Hemodial Int. 2004;2:188-92.

4. Ikizler TA, Kliger AS. Minimizing the risk of COVID-19 among patients on dialysis. Nat Rev Nephrol Nat Publ Group. 2020;16:1-3.

5. Scarpioni R, Manini A, Valsania T, De Amicis S, Albertazzi V, Melfa L, et al. Covid-19 and its impact on nephropathic patients: the experience at Ospedale "Guglielmo da Saliceto" in Piacenza. G Ital Nefrol. 2020;37:2020.

6. Goicoechea M, Sánchez Cámara LA, Macías N, Muñoz de Morales A, González Rojas Á, Bascuñana A, et al. COVID-19: clinical course and outcomes of 36 maintenance hemodialysis patients from a single center in Spain. Kidney Int Elsevier. 2020;98:27.

7. Registry R. COVID-19 surveillance report for renal centres in the UK - up to 6th may 2020; 2020.

8. Meyer TW, Hostetter TH, Watnick S. Twice-weekly hemodialysis is an option for many patients in times of dialysis unit stress. J Am Soc Nephrol. 2020; 31(6):1141.

9. Mehrotra R. Counterpoint: twice-weekly hemodialysis should be an approach of last resort even in times of dialysis unit stress. J Am Soc Nephrol. 2020;31(6):1143.

10. Fotheringham J, Fogarty DG, El Nahas M, Campbell MJ, Farrington K. The mortality and hospitalization rates associated with the long interdialytic gap in thrice-weekly hemodialysis patients. Kidney Int. 2015;88:569-75.

11. Foley RN, Gilbertson DT, Murray T, Collins AJ. Long interdialytic interval and mortality among patients receiving hemodialysis. N Engl J Med. 2011;365: 1099-107.

12. NICE. Sodium zirconium cyclosilicate for treating hyperkalaemia. Technol Apprais Guid[TA599]. NICE. 2019;372(3):222.

13. BiCARB study group. Clinical and cost-effectiveness of oral sodium bicarbonate therapy for older patients with chronic kidney disease and lowgrade acidosis (BiCARB): a pragmatic randomised, double-blind, placebocontrolled trial. BMC MedSpringer. 2020;18:1-16.

14. Green D, Roberts $P$, New D, Kalra P. Sudden cardiac death in hemodialysis patients: an in-depth review. Am J Kidney Dis. 2011;57:921-9.

15. Charra B, Chazot C, Jean G, Hurot JM, Vanel T, Terrat JC, et al. Long $3 \times 8$ hr dialysis: a three-decade summary. J Nephrol. 2003;16:S64-9.

16. Kotanko P, Garg AX, Depner T, Pierratos A, Chan CT, Levin NW, et al. Effects of frequent hemodialysis on blood pressure: results from the randomized frequent hemodialysis network trials. Hemodial Int NIH Public Access. 2015; 19:386-401.

17. Blankestijn PJ, Ligtenberg G. Volume-independent mechanisms of hypertension in hemodialysis patients: clinical implications. Semin Dial. 2004;17:265-9.
18. Chang TI. Systolic blood pressure and mortality in patients on hemodialysis. Curr Hypertens Rep NIH Public Access. 2011;13:362-9.

19. Yusuf AA, Hu Y, Singh B, Menoyo JA, Wetmore JB. Serum potassium levels and mortality in hemodialysis patients: a retrospective cohort study. Am J Nephrol S Karger AG. 2016;44:179-86.

20. Ferrey A, You AS, Kovesdy CP, Nakata T, Veliz M, Nguyen DV, et al. Dialysate potassium and mortality in a prospective hemodialysis cohort. Am J Nephrol NIH Public Access. 2018;47:415.

21. Agrawal D, Agrawal D, Tripathi N, Segal M. Potassim profiling in hemodialysis. J Renal Hepatic Disord. 2018;2(2):6-9.

22. Ashby D, Borman N, Burton J, Corbett R, Davenport A, Farrington K, et al. Renal association clinical practice guideline on Haemodialysis. BMC Nephrol BioMed Central. 2019;20:379.

\section{Publisher's Note}

Springer Nature remains neutral with regard to jurisdictional claims in published maps and institutional affiliations.
Ready to submit your research? Choose BMC and benefit from:

- fast, convenient online submission

- thorough peer review by experienced researchers in your field

- rapid publication on acceptance

- support for research data, including large and complex data types

- gold Open Access which fosters wider collaboration and increased citations

- maximum visibility for your research: over $100 \mathrm{M}$ website views per year

At BMC, research is always in progress.

Learn more biomedcentral.com/submissions 